\title{
Current status and research activity at the INRNE cyclotron laboratory
}

\author{
Anguel Demerdjiev ${ }^{1, *}$, Dimitar Tonev ${ }^{1}$, Nikolay Goutev ${ }^{1}$, Galina Asova ${ }^{1}$, and Ary Artinyan ${ }^{1}$ \\ ${ }^{1}$ Institute for Nuclear Research and Nuclear Energy, Bulgarian Academy of Sciences, Tzarigradsko chaussee 72, Blvd., BG-1784, \\ Sofia, Bulgaria
}

\begin{abstract}
A review and update on the status of the Cyclotron Physics Laboratory at the Institute for Nuclear Research and Nuclear Energy as well as related research activity is presented.

The main part of the research activities at the Cyclotron Physics Laboratory is directed to simulations for design considerations, for the cyclotron vault for some of the target radioisotopes. In this paper, a review on the simulations, conducted at the laboratory, related to ${ }^{18} \mathrm{~F}$ production is included.

A model for studying the effect of attenuation and absorption of neutron radiation, emitted by a point source with fixed energy of $14.1 \mathrm{MeV}$ is developed. Preliminary results from this study are shown.
\end{abstract}

\section{Introduction}

The National Cyclotron Center project was launched, as an initiative of the Council of Ministers of Republic of Bulgaria and the management of the Institute for Nuclear Research and Nuclear Energy (INRNE), in 2012 with the purpose to solve two problems on national level. The first is the shortage of domestic short-lived medical radioisotopes. And, since, presently Bulgaria has no nuclear research facility or an accelerator this project is an opportunity to restart our experimental programme in the field of nuclear physics and it will also contribute to many interdisciplinary fields related to it.

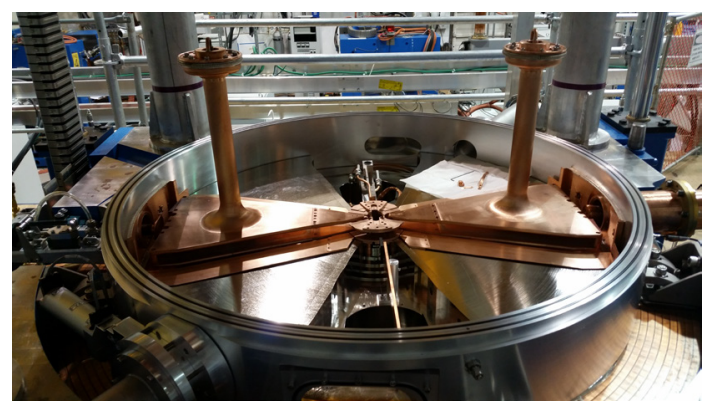

Figure 1. A picture of the open cyclotron TR-24 during the FAT in Vancouver in August 2015.

For this purpose, a new cyclotron laboratory is planned to be built as part of INRNE. It will consist of a specialized building that meets the regulatory requirements of radiation safety and GMP (good manufacturing practices) in the pharmaceutical industry and a vault for the T-24 cyclotron. The laboratory will be divided into two sectors: one dedicated to research and development in the fields of natural sciences and radiopharmaceuticals with strong

*e-mail: angeld@inrne.bas.bg educational functions; second one - purely practical - for production of ${ }^{18} \mathrm{~F}$-FDG and eventually other radiopharmaceuticals [1].

Currently, the research activities related to the cyclotron at INRNE are directed to numerical studies on the possibilities to produce various medical isotopes and radiological characterization of the setup. Our effort is dedicated to the production of ${ }^{18} \mathrm{~F}$, since in Bulgaria ${ }^{18} \mathrm{~F}-\mathrm{FDG}$ and ${ }^{99 m}$ Tc have highest shares in the nuclear medicine procedures with a constant rise of the first.

Recently obtained preliminary results from a model, developed for comparing the absorbing and attenuating properties of neutron radiation of different materials, are going to be presented. Here we considered a boron enriched concrete and low density ordinary concrete.

In parallel, a new possible research activity is considered - development of plasma sources for additional proton beam acceleration [2].

\section{The TR-24 cyclotron}

In January 2016, at INRNE, after the successful factory acceptance tests (FAT) in August 2015 (carried out in Vancouver, Canada), was delivered a TR-24 cyclotron (figure 1). It is constructed by Advanced Cyclotron Systems Inc. A proton beam with variable energy $(15-24 \mathrm{MeV})$ is produced based on the acceleration of negative hydrogen ions extracted from an external CUSP ion source. The beam is of high quality and current - up to $400 \mu \mathrm{A}$, upgradeable to $1 \mathrm{~mA}$.

There are four ports for beam extraction and the cyclotron allows the simultaneous extraction of two. With such parameters of the proton beam the cyclotron is capable to produce more than 15 radioisotopes by irradiating simultaneously two high current targets placed at the exit ports. Thus, additionally were purchased one Y-like beam 


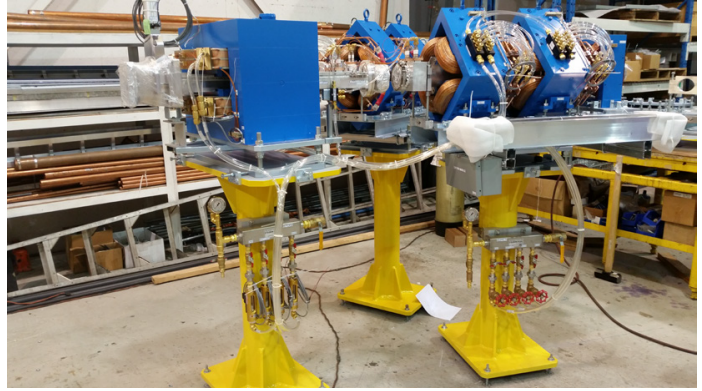

Figure 2. INRNE cyclotronY-line

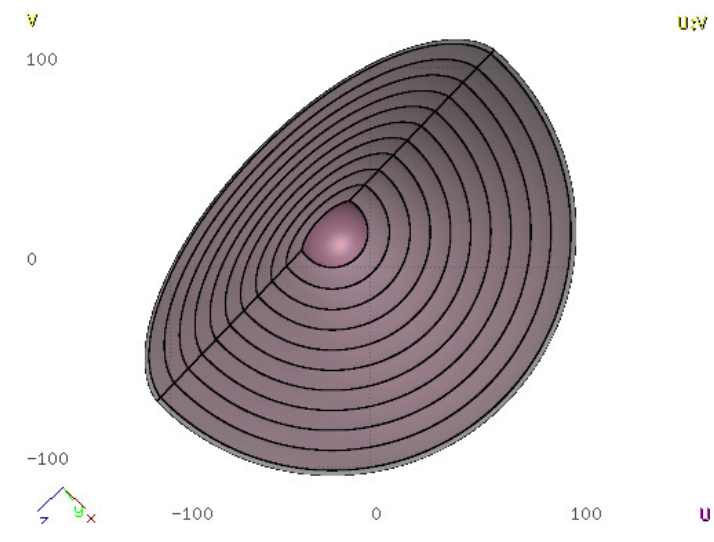

Figure 3. Schematic representation of the model. The axis dimensions are in $\mathrm{cm}$. The neutron source is positioned in the geometrical center of the sphere.

line (figure 2), two target stations and three high current liquid targets for production of ${ }^{18} \mathrm{~F}$.

\section{Current status of INRNE Cyclotron Physics Laboratory and research activities}

A well-established practice for target and shielding design and activation analysis is employing the Monte-Carlo approach based on numerical simulations using the FLUKA tool $[3,4]$. It uses experimentally measured cross sections. The whole process for radiological characterization of the vault and the local target shielding could be systematized in two steps: first - to simulate target irradiation and to assess the secondary particles; second - using the secondary particles as source irradiating components of the: vault; local target shielding. A detailed model of the delivered 3.8 $\mathrm{mL}$ liquid targets for production of ${ }^{18} \mathrm{~F}$ was made. And the first important step was done - obtaining collision tape with the emitted secondary particles from irradiated target for production of ${ }^{18} \mathrm{~F}$. The just reviewed previous studies $[5,6]$ have shown that local shielding around target filled with ${ }^{18} \mathrm{O}$-enriched water will be useful in order to limit the spatial distribution of the secondaries, to decrease the activation of components within the vault and, thus, to increase the lifetime of the facility, and to decrease the effective dose of the personnel.
In the current study, we simulated a point source, positioned in the center of an air filled sphere, surrounded by a concrete spherical shell, emitting neutrons, with fixed energy of $14.1 \mathrm{MeV}$ (figure 3). We checked the reaction rate decrease in depth of the spherical shell for different materials. The model and the preliminary results are discussed in the next section.

The other direction of our effort is directed to development of a plasma source for additional acceleration of proton beam. In the study, we applied a fluid model to a microwave plasma source exemplifying a plasma filled array of cavities made of a dielectric material with high permittivity. We have obtained preliminary results for the distribution of the $\varphi$ component of the electric field and plasma parameters (electron density and temperature) in the discharge. An estimation of the maximum electric field which could be waked inside the simulated discharge was also made. This is already published and discussed in [2].

\section{Preliminary studies on the absorption of neutron point source radiation}

\subsection{Description of the model}

For description of the model, a slice of the threedimensional view of the simulated modeling domain is shown on figure 3. A simplified scenario is considered - a neutron source positioned close to the vault walls. We studied a point source emitting neutrons with fixed energy of $14.1 \mathrm{MeV}$ placed in the center of an air filled sphere with radius of $20 \mathrm{~cm}$. The air sphere is surrounded by concrete spherical shell with thickness $123 \mathrm{~cm}$. On every $10 \mathrm{~cm}$ are placed two activation foils (the first pair is on the inner concrete shell surface $)-a{ }^{93} \mathrm{Nb}(\mathrm{n}, 2 \mathrm{n}){ }^{92 m} \mathrm{Nb}$ with thickness of $0.1 \mathrm{~cm}$ sensitive to neutron above $10 \mathrm{MeV}$ and ${ }^{197} \mathrm{Au}(\mathrm{n}, \gamma){ }^{198} \mathrm{Au}$ - sensitive to low-energy neutron, reactions.

In the foils, we are scoring the number of reactions per primary neutron normalized to the volume of the respective foil - reaction rate. We tested two different concretes (table 1) - low-activation Boron enriched concrete (BEC) [7] and a ordinary concrete (OC) [7].

The obtained results are for neutrons with $14.1 \mathrm{MeV}$ and irradiation time of 7 hours.

\subsection{Results and discussions}

In figures 4, 5 are presented the calculated reaction rates of ${ }^{197} \mathrm{Au}(\mathrm{n}, \gamma){ }^{198} \mathrm{Au}$ and ${ }^{93} \mathrm{Nb}(\mathrm{n}, 2 \mathrm{n}){ }^{92 m} \mathrm{Nb}$, as a function of depth from the inner surface of the concrete shell.

As expected in the case of BEC, since Boron has good attenuating and absorbing properties for slow neutrons (figure 4) on overall the reaction rate is decreased by four orders of magnitude $\left(10^{-5}-10^{-9}\right)$. While in the case of OC about one order of magnitude $\left(10^{-4}-10^{-5}\right)$.

In the case of neutrons with an energy above $10 \mathrm{MeV}$, shown in figure 5, the behavior of the reaction rate for the two cases is similar. It is decreased by two orders of magnitude in the first $40 \mathrm{~cm}$. 
Table 1. Density and weight percent of each element in BEC and $\mathrm{OC}$

\begin{tabular}{lll}
\hline & BEC & OC \\
Density $\left(\mathrm{g} / \mathrm{cm}^{3}\right)$ & 2.0 & 2.1 \\
\hline Weight percent $(\%)$ & & \\
$\mathrm{H}$ & 2.88 & 1.04 \\
$\mathrm{~B}$ & 10.4 & - \\
$\mathrm{C}$ & - & 0.1 \\
$\mathrm{O}$ & 60.4 & 54.6 \\
$\mathrm{Na}$ & 0.03 & - \\
$\mathrm{Mg}$ & 0.37 & 0.23 \\
$\mathrm{Al}$ & 6.31 & 3.49 \\
$\mathrm{Si}$ & 0.66 & 34.7 \\
$\mathrm{P}$ & 0.02 & - \\
$\mathrm{S}$ & 0.04 & - \\
$\mathrm{K}$ & 0.13 & - \\
$\mathrm{Ca}$ & 18.2 & 4.47 \\
$\mathrm{Ti}$ & 0.31 & - \\
$\mathrm{Mn}$ & 0.01 & - \\
$\mathrm{Fe}$ & 0.27 & 1.43 \\
\hline
\end{tabular}

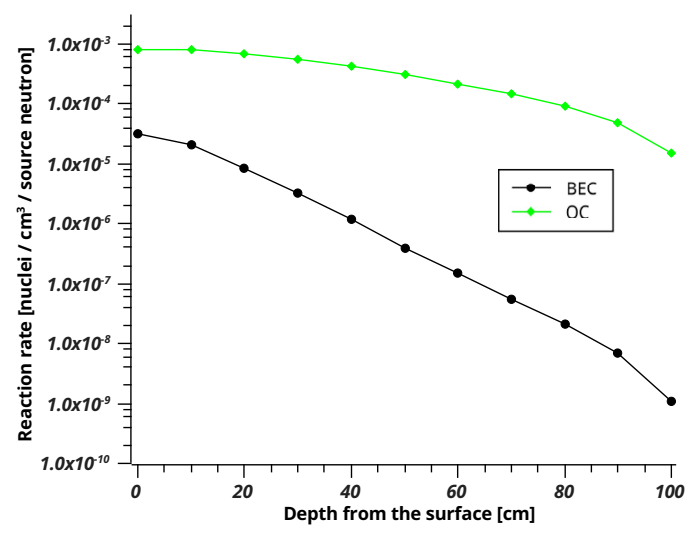

Figure 4. Calculated reaction rates of ${ }^{197} \mathrm{Au}(\mathrm{n}, \gamma){ }^{198} \mathrm{Au}$ reaction in $\mathrm{BEC}, \mathrm{OC}$

An evaluation of the attenuation of the neutron flux in the first $100 \mathrm{~cm}$ of the concrete is made by calculating

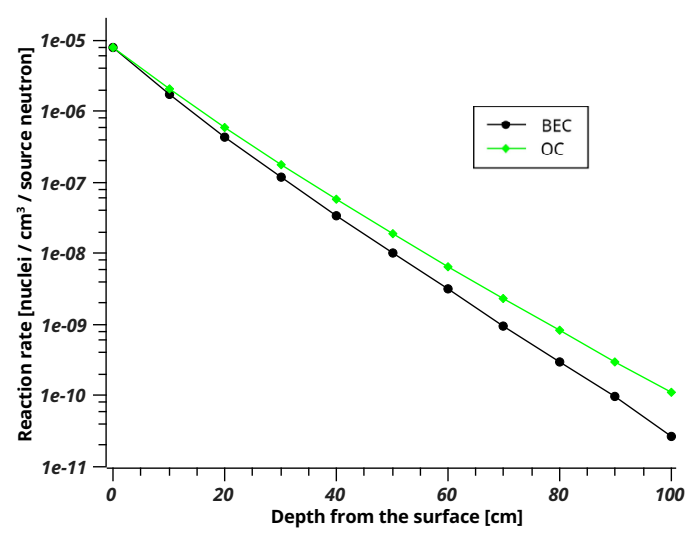

Figure 5. Calculated reaction rates of ${ }^{93} \mathrm{Nb}(\mathrm{n}, 2 \mathrm{n}){ }^{82 m} \mathrm{Nb}$ reaction in $\mathrm{BEC}, \mathrm{OC}$ the reaction rate. The further possible step is to change the point source with the already obtained source of secondary particles [5] and to test recipes for concretes consisting of materials which are available in Bulgaria.

\section{Conclusion}

The current research activity related to the cyclotron project at INRNE is directed to vault and local target shielding design. A model for calculating the reaction rate, simulating activation foils is made. Further work is required to complete the vault and local target shielding design.

The research has been supported by Bulgarian Science Fund under Contract No. 08/6, 13.12.2016.

\section{References}

[1] D. Tonev, N. Goutev, L. S. Georgiev, J. Phys. Conf. Proc., 724, 012049 (2016)

[2] A. Demerdjiev, N Goutev, D. Tonev, J. Phys. Conf. Proc., 1023, 012033 (2018)

[3] T. T. Böhlen, F. Cerutti, M. P. W. Chin, A. Fassò, A. Ferrari, P. G. Ortega, A. Mairani, P. R. Sala, G. Smirnov, A. Vlachoudis, Nuclear Data Sheets, 120, $211-214$ (2014)

[4] A. Ferrari, P. R. Sala, A. Fassò, J. Ranft, FLUKA: A multi-particle transport code (program version 2005), (CERN-2005-010, INFN-TC-05-11, SLACR-773, 2005)

[5] M. Yavahchova, G. Asova, N. Goutev, D. Tonev, Science and Technologies, VI, 3 (2016)

[6] G. Asova, N. Goutev, D. Tonev, A. Artinyan, J. Phys.: Conf. Series, 1023, 012008 (2018)

[7] Satoshi Sato, Chikara Konno, Hiroshi Nakashima, Ryosuke Shionaga, Hiroyuki Nose, Yuji Ito, Hirohide Hashimoto, J. Nucl. Sci. Technol., 55, 410 - 417 (2018) 